\title{
Verlust der Ethik? Bioethik zwischen Institutionalisierung und Ideologiekritik
}

_Originalartikel

a Departement für Moraltheologie und Ethik, Universität Fribourg

\section{Zusammenfassung_English and French abstracts see p. 15}

Sowohl die Institutionalisierung der biomedizinischen Ethik als auch darauf reagierende ideologiekritische Stellungnahmen sind Ausdruck gesellschaftlicher Verunsicherungen und Unzufriedenheiten, die u. a. aufgrund unübersichtlicher und rascher Fortschritte in der Biomedizin, der Auflösung stabiler Werteund Orientierungssysteme, dem Autoritätsverlust der Ärzteschaft und der Betonung der Autonomie entstehen. Dabei droht, so die hier vertretene These, das selbstkritische Nachdenken, das kluge Abwägen von Für und Wider auf der Basis von Erfahrungen, moralischen Überzeugungen, verbreiteten Werthaltungen, plausiblen Argumenten und ethischen Theorien zu erodieren. Im Anschluss an eine kurze Bestandsaufnahme werden Beispiele für den vorschnellen Abbruch ethischer Suchprozesse geschildert, die heute mit Bezug auf das geltende Recht, die Autonomie der Betroffenen, fehlende empirische Daten, einzuhaltende Prozeduren, kulturell oder religiös hochgehaltene Traditionen oder auch ideologiekritische Bedenken begründet werden. Abschliessend werden Bedingungen für eine gute und zukunftsfähige Bio- und Medizinethik entworfen: Wünschenswert wäre, auch in Zukunft Räume zu bewahren, in welchen kontroverse, interdisziplinäre und auf ethische Erkenntnis hin angelegte Suchprozesse möglich bleiben. Ein realistisches Ziel besteht darin, dass an die Stelle eines diffusen moralischen Pluralismus ein aufgeklärter Pluralismus vernünftig begründeter Meinungen tritt, der moralische Orientierung und verantwortliches Handeln ermöglicht.

Key words: Bioethik; Medizinethik; ethischer Diskurs; Institutionalisierung; Ideologiekritik

\section{Gefährdete Ethik?}

Nie war Ethik so gefragt wie heute, nie war sie derart präsent in Öffentlichkeit, Medien, Politik und Recht. Das gilt in besonderem Masse für die biomedizinische Ethik oder Bioethik, die gegenwärtig in der Schweiz beispielsweise bei der Einführung der Patientenverfügungen im Erwachsenenschutzrecht, der Aufhebung des Verbots der Präimplantationsdiagnostik im Fortpflanzungsmedizingesetz, der Neuregelung der Suizidbeihilfe im Strafgesetzbuch oder bei der Ausgestaltung eines neuen Humanforschungsgesetzes gefragt ist.

Seit einigen Jahren ist ein Institutionalisierungsprozess von Ethik, ethischer Beratung und Ausbildung nicht nur in Spitälern und Heimen, sondern auch in universitären, politischen und kirchlichen Institutionen im Gang, der sich in den nächsten Jahren noch verstärken dürfte. Gleichzeitig melden sich skeptische bis ideologiekritische Stimmen zu Wort, welche die damit verbundene Etablierung, Politisierung und Verrechtlichung der Ethik ablehnen: Der Institutionalisierungsprozess trage lediglich dazu bei, von den wirklichen Problemen, namentlich der Auflösung traditionell verankerter Werthaltungen und der gelebten Moral abzulenken, er sei Teil biopolitischer Diskurse und Praktiken, die auf die Regulierung des Lebens zielten oder leiste Geburtshelferdienste zugunsten ökonomischer Interessen der Pharma- und Gesundheitsindustrie. In einem Interview von 2009 meinte der damalige Bundesrat Pascal Couchepin auf die Frage, wozu wir
Ethikkommissionen brauchen: Er habe den Eindruck, diese Kommissionen würden die Menschen beruhigen; anstatt sich selbst zu fragen, was ethisch sei, habe man heute eine Kommission, die das für einen erledige (1; kritisch dazu 2). Ironische, skeptische und ideologiekritische Interventionen unterscheiden sich insofern vom ethischen Diskurs, als es ihnen nicht um das bessere Argument, um Chancen und Gefahren einer zu bewertenden Handlungsweise oder Technik geht, sondern - gleichsam auf einer Metaebene - um das Beklagen von Werteverlust, das Aufdecken von Interessenkonflikten und Aporien, die Warnung vor falschen Weltanschauungen oder auch vor Bevormundung.

Sowohl die Institutionalisierung als auch die Ideologiekritik sind Ausdruck gesellschaftlicher Verunsicherungen und Unzufriedenheiten, die u. a. aufgrund unübersichtlicher und rascher Fortschritte in der Biomedizin, der Auflösung stabiler Werte- und Orientierungssysteme, dem Autoritätsverlust der Ärzteschaft und der Betonung der Autonomie, nicht zuletzt aus Ängsten vor Ausweitungs- und Missbrauchsphänomenen im Umgang mit dem menschlichen Leben entstehen. Auch wenn sowohl die Institutionalisierung als auch die Ideologiekritik ihre positiven Seiten haben, droht dabei, so meine These, das selbstkritische Nachdenken, das kluge Abwägen von Für und Wider auf der Basis von Erfahrungen, moralischen Überzeugungen, verbreiteten Werthaltungen, plausiblen Argumenten und ethischen Theorien zu erodieren. Gefährdet 
scheinen insbesondere Ethikdispute zu sein, die ergebnisoffen begonnen werden und als suchende Diskurse angelegt sind. Wie wichtig diese aber sind, zeigt sich heute angesichts der Reprogrammierbarkeit somatischer Zellen und der Frage, was unter einem Embryo verstanden werden soll, angesichts neuer Möglichkeiten der Gendiagnostik und der Frage, was ein Recht auf Nichtwissen noch beinhalten kann, oder angesichts der Suche nach Kriterien zur Bestimmung sinnloser Behandlungen bei schwer Kranken und Sterbenden. Offenkundig genügen bestehende (Stammzellforschungsgesetz und Bundesgesetz über genetische Untersuchungen beim Menschen) oder geplante (Initiativen zur rechtlichen Regulierung von Entscheidungen zum Behandlungsabbruch und -verzicht) rechtliche Regelungen alleine nicht, um angesichts komplexer Veränderungen Orientierung zu schaffen.

Ethische Diskurse ergebnisoffen anzugehen heisst nicht, dass die Beteiligten keine dezidierten ethischen Standpunkte vertreten sollten. Im Gegenteil:Je reflektierter die vertretenen Ethikansätze und je grösser die Bereitschaft, die soziokulturellen Hintergrundannahmen, Menschen-, Welt- und unter Umständen auch Gottesbilder transparent zu machen, desto eher lassen sich kontroverse, interdisziplinäre und auf ethische Erkenntnis hin angelegte Suchprozesse realisieren bzw. - das sei angesichts verbreiteter non-kognitivistischer und fundamentalistischer Ethikansätze ebenfalls betont - die Grenzen ethischer Verständigungsmöglichkeit bestimmen $(3,4)$. Erst der gegenseitige Respekt aller am Diskurs Beteiligter ermöglicht vertiefende, sinnerschliessende Ethikdiskurse, die sich von rein strategischen Debatten unterscheiden. Nötig ist eine Ethik als Reflexion auf Moral, welche tugend- und güterethische Überlegungen einbezieht und bei gelebten moralischen Überzeugungen ansetzt, sich also nicht auf die reine Normativität von Aussagen beschränkt (5). Wie wichtig diese erweiterte Ethikperspektive ist, zeigen beispielsweise Diskussionen über die unfreiwillige Kinderlosigkeit und einen verantwortlichen Umgang mit den Möglichkeiten der modernen Reproduktionsmedizin (6).

\section{Ethikdispute vorzeitig beenden?}

Vorzeitiges Abbrechen ethischer Suchprozesse ist an verschiedenen Orten zu beobachten und wird unterschiedlich begründet: mit Bezug auf das geltende Recht, die Autonomie der Betroffenen, fehlende empirische Daten, einzuhaltende Prozeduren, kulturell oder religiös hochgehaltene Traditionen oder ideologiekritische Bedenken. Aus meiner Sicht als katholischer Theologe und Ethiker, der seit Jahren an bioethischen Auseinandersetzungen - auch an Institutionalisierungsprozessen und der Bioethik-Kritik - in der Schweiz beteiligt ist (7), möchte ich einige Beispiele herausgreifen, die durchaus auch selbstkritisch zu verstehen sind.

Aufschlussreich ist die gegenwärtige Auseinandersetzung um die Bezeichnung und Funktion von Forschungsethikkommissionen. Im Zusammenhang mit dem Entwurf des neuen Humanforschungsgesetzes wird in der Schweiz darüber debattiert, ob die besagten Kommissionen wie bis anhin als Ethikkommissionen oder neu als Kontrollkommissionen bezeichnet werden sollten. Zentral ist die Frage, ob die Auf- gabe dieser Kommissionen darin besteht, die Einhaltung rechtlicher Normen zu prüfen, oder ob es darüber hinaus auch um die Überprüfung ethischer Normen gehen soll. Da die Kommissionen rechtlich verbindliche Entscheidungen treffen, ist die Klärung der einzuhaltenden Normen von grösster Bedeutung sowohl für die Forscherinnen und Forscher als auch die Glaubwürdigkeit des Rechtssystems. Beide Varianten stellen letztlich Kompromisse dar: Werden die Kommissionen in Zukunft als Kontrollkommissionen bezeichnet, fällt nur noch die rechtliche Überprüfung von Forschungsprotokollen in ihren Aufgabenbereich; werden sie weiterhin Ethikkommissionen genannt, bleibt eine gewisse Rechtsunsicherheit bestehen, insofern sich rechtlich nicht eindeutig bestimmen lässt, was in einem ethischen Überprüfungsverfahren genau gewichtet werden soll. Obgleich auch in einem rechtlichen Prüfverfahren in jedem Einzelfall ein Interpretationsprozess nötig wird, ist es nicht erstaunlich, dass sich viele Juristinnen und Juristen gegen die Ethik im Namen der Kommissionen ausgesprochen haben, während eine 2009 durchgeführte öffentliche Vernehmlassung das Gegenteil ergeben hat. Was hier geschieht, ist - neben einer spannenden Debatte über das Verhältnis von Ethik und Recht - eine Folge des Verrechtlichungsprozesses von Ethik. Dadurch, dass Ethikkommissionen nicht nur beraten, sondern rechtlich verbindliche Entscheidungen zu fällen haben, erhalten sie gesundheitspolizeiliche Aufgaben, können sie haftungsrechtlich belangt werden und sind rechtliche Rekurse gegen ihre Entscheide möglich. Die Ethik begibt sich hier gleichsam freiwillig in ein Korsett rechtlich vorgegebener Normen, um in heiklen Entscheidungsprozessen präsent bleiben zu können.

Problematisch sind zudem Selbstbeschränkungen, die sich die Bioethik mit Hinweis auf das Autonomieprinzip selbst auferlegt. Steht bei einer Entscheidung für die Betroffenen viel auf dem Spiel - zu denken wäre an die Beteiligung an einem genetischen Test oder eine Lebendleberspende - stellt sich die Frage, gemäss welcher Kriterien die Einzelnen sich entscheiden sollten. Obgleich der auf den Einzelnen lastende Entscheidungsdruck im Zeichen der Reflexivität der Moderne in den letzten Jahren grösser wurde, beschränken sich bioethische Diskurse weitgehend auf die Betonung der individuellen Autonomie im Sinne der negativen Freiheit und verpassen es, Modelle gelungener Entscheidungen einzubeziehen. So wird in den Debatten um die Patientenverfügungen über den Verpflichtungsgrad von Verfügungen bzw. deren Reichweite gestritten, während Fragen darüber, was sich der Einzelne beispielsweise für den Fall eines irreversiblen Wachkomas sinnvoller Weise wünschen sollte, weitgehend unbeachtet bleiben. Die im Zusammenhang mit möglichen finanziellen Einsparungen bei der Versorgung Sterbender geäusserte Idee eines Schweizer Parlamentariers, die Abfassung einer Patientenverfügungen für alle Bürgerinnen und Bürger ab sechzig Jahren verpflichtend zu machen, bringt diese Einseitigkeit auf den Punkt: Im Namen des Selbstbestimmungsrechts sollen den Einzelnen Aufgaben auferlegt werden, die zur Lösung gesellschaftlicher Probleme wie den steigenden Gesundheitskosten bei- 
tragen sollen. Auf diese Weise würden im Namen der Freiheit neue Zwänge geschaffen, die sich einem einseitig an der Machbarkeit orientierten Menschenbild verdanken (8).

Die gegenwärtige Vorliebe der Medizinethik für die sozialempirische Forschung (9) wirft ebenfalls Fragen zu einem womöglich vorschnellen Abbruch ethischer Reflexion auf. Zu begrüssen ist die Erfahrungsnähe und das starke Interesse an den Ergebnissen der empirischen Sozialforschung, skeptischer zu beurteilen ist die Tendenz, sozialempirische Forschung selbst als Ethik zu bezeichnen, als unzureichend abzulehnen aber wäre der Ansatz, die Ethik sollte sich darin erschöpfen. Häufig werden durch die Ergebnisse qualitativer Interviews beispielsweise mit Eltern, die ihre überzähligen Embryonen der Forschung zur Verfügung stellen, oder auch quantitativer Untersuchungen, z. B. zu Entscheidungen am Lebensende, ethische Kontroversen erst an den entscheidenden Punkten eröffnet, in den seltensten Fällen jedoch werden sie dadurch beantwortet. Naheliegend ist daher eine sinnvolle Arbeitsteilung, in welcher die biomedizinische Ethik auf Ergebnissen der Human-, Sozial- und Naturwissenschaften aufbaut, auch an den Forschungsanlagen mitarbeitet, sich aber nicht auf diese beschränkt, sondern auf dieser Basis wichtige Fragestellungen weiterverfolgt (10, S. 256).

Im Bereich der klinischen Ethik einschlägig sind zudem die Entwicklung und Anwendung prozeduraler Entscheidfindungsmodelle. Zweifellos gehören solche Verfahren heute zu anerkannten und bewährten bereichsethischen Methoden, die zur Entlastung in Einzelsituationen und zur ethischen Orientierung bei institutionellen Entscheidungen wesentlich beitragen können (11). Im Sinne der auch hier möglichen Selbstbescheidung der Ethik werden sie jedoch dann problematisch, wenn Prozeduren derart detailliert geregelt und in Einzelschritte zerlegt werden, dass normative und evaluative Diskussionen zu Alibiübungen werden oder gänzlich verschwinden. Kommt es soweit, lassen sich klinische Ethikrunden nicht mehr von anderen Fallbesprechungen oder Supervisionen unterscheiden; mit der ethischen Auseinandersetzung verschwindet dann der eigentliche Sinn dieser oft zeitintensiven Foren.

Ähnliche Effekte können sich auch aus der falsch verstandenen Idee ergeben, schwierige Dilemmaentscheidungen liessen sich im Sinne einer "Auslagerung des Gewissens» (12) an Ethikgremien delegieren. Dass solche Erwartungen enttäuscht werden müssen, zeigen die Konflikte, die im Frühjahr 2009 zwischen Bundesrat Pascal Couchepin und der Nationalen Ethikkommission (NEK) deutlich wurden. In einem Interview mit der deutschen Wochenzeitschrift «Die Zeit» äusserte sich der damalige Gesundheitsminister enttäuscht zur Arbeit seiner Kommission: «Die Ethik-Kommissionen sind in ganz Europa ein Problem. Nicht weil sie uns sagen, was wir wollen, sondern weil sie uns sagen, was sie wollen - aufgrund von Kriterien, die sehr oft der Mehrheitsmoral folgen.» Und weiter: "Am Anfang hatte man gedacht, diese Leute werden die moralischen Aspekte einer Sache beleuchten. Aber sie machen heute manchmal grosse Berichte, in denen steht: Was modern ist, ist richtig. Das ist ein Problem.»(1) Offensichtlich hatte er eine modernitätskritische Ethikkommission erwartet, während die real existierende NEK vergleichbar mit der
«Gesellschaft oder dem Parlament im Kleinen» im Sinne der auch dort bestehenden Mehrheitsmoral votierte. Die sich hier manifestierende Politisierung von Ethikgremien und deren Empfehlungen zeigt sich nicht nur daran, dass Ratschläge von Ethikkommissionen in Form von Minderheits- und Mehrheitsvoten abgegeben werden, sondern auch insofern, als die politisch Verantwortlichen versuchen, den Inhalt ethischer Empfehlungen über die Ernennung von Kommissionsmitgliedern in ihrem Sinne zu beeinflussen (13). Eine der ersten Amtshandlungen von Präsident Barak Obama bestand darin, die von seinem Vorgänger eingesetzte Bioethikkommission zu entlassen; erst im November 2009 hat er eine seinen Vorstellungen entsprechende neue Kommission einberufen.

Auch in weltanschaulich und religiös gebundenen Ethiken kann es zu vorzeitigen Abbrüchen der Auseinandersetzungen kommen, wenn sich beispielsweise - wie im Fall der Stammzelldebatten - die nahezu gesamte Aufmerksamkeit auf einen bestimmten Aspekt der Diskussion richtet, wie in der katholischen Moraltheologie auf die Zerstörung menschlicher Embryonen. Dass die Stammzellforschung durch die kürzlich gelungene Reprogrammierung von ausdifferenzierten Körperzellen zu induzierten pluripotenten Stammzellen, aus welchen sich womöglich wiederum «Embryonen» herstellen lassen, neue Grenzfragen aufwerfen, kam aufgrund entsprechender Erfolge im Tierversuch nicht überraschend (14). Jenseits und unabhängig von der umstrittenen Embryonenforschung werden in der Zellbiologie dadurch heute Grenzüberschreitungen möglich, welche neue ethische Fragen aufwerfen, namentlich nach Orientierungsmöglichkeiten an der menschlichen Natur (15), die auch aus moraltheologischer Perspektive noch gründlich durchdacht werden müssen (16).

Besondere Herausforderungen ergeben sich nicht zuletzt in Auseinandersetzung mit den erwähnten ideologiekritischen Beiträgen zur Bioethik, deren Ziel u. a. darin besteht, die bioethischen Debatten als Teil von Machtdiskursen zu überführen (18). Diese häufig von Michel Foucaults Diskurstheorie und seinem Begriff der Biomacht inspirierten Überlegungen verlaufen quer zu den üblichen bioethischen Debatten, beschäftigen sich beispielsweise mit erkenntnistheoretischen Metadiskursen über die philosophischen Grundbegriffe Natur und Leben, setzen sich mit der Biologisierung des Sozialen sowie der menschlichen Natur auseinander, dekonstruieren die Idee eines autonomen Handlungssubjekts oder suchen auf neovitalistische Weise Orientierung an einem kollektivistisch verstandenen Lebensbegriff (17).

\section{Zukunft der Bioethik?}

Es wäre wünschenswert, in der Bio- und Medizinethik auch in Zukunft Räume zu bewahren, in welchen kontroverse, interdisziplinäre und auf ethische Erkenntnis hin angelegte Suchprozesse möglich sind. Dabei sollten im Sinne des ethischen Kohärentismus, der sowohl konkrete Werthaltungen (Intuitionen) als auch ethische Basisprinzipien berücksichtigt, performative, regulative, theoretische, kulturelle und durchaus auch ideologiekritische Ethikexpertisen mit einbe- 
zogen werden, um der Komplexität der Problemstellungen angemessen begegnen zu können (19). Damit eine solche zukunftsfähige Bioethik möglich werden kann, wären etwa folgende Bedingungen zu erfüllen: Wichtig wäre eine heute knappe Ressource, nämlich die nötige Zeit, um komplexe Fragen durchdringen zu können. Auseinandersetzungen sollten zudem interdisziplinär angegangen werden, um einerseits die Expertisen, andererseits aber auch verschiedene professionelle Perspektiven berücksichtigen zu können. Wesentlich ist zudem die Erfahrungsnähe, insofern sich die entscheidenden ethischen Fragen in der Regel in Auseinandersetzung mit der Praxis ergeben. Inhaltlich sollte die globale Perspektive und damit diejenige der wirtschaftlich benachteiligten Länder stets mitgedacht werden. Persönlich wünschen würde ich mir zudem Beteiligte, die einen kontextsensitiven ethischen Ansatz vertreten, sowohl Aspekte des Richtigen als auch des Guten mit in die Überlegungen einfliessen lassen, an Überzeugungen und Werthaltungen anzuknüpfen versuchen und die gleichzeitig von der Begründbarkeit moralischer Ansprüche überzeugt sind, also ethische Kognitivisten sind. In der Gewissheit, dass diese Wünsche nicht immer erfüllt werden, halte ich es für eine weitere Bedingung einer guten Medizinund Bioethik, dass die unterschiedlichen Ethikansätze und Hintergrundannahmen möglichst transparent gemacht werden; damit dies gelingen kann, gilt wiederum die Bedingung des gegenseitigen Respekts voreinander, der davon ausgeht, dass in der Gesellschaft unterschiedliche Überzeugungssysteme nebeneinander existieren, die in sich kohärent begründet sein können. Darum besteht in der bescheidenen Zurückhaltung hinsichtlich Letztbegründungsansprüchen und abschliessender Urteile eine Basistugend einer guten und zukunftsweisenden biomedizinischen Ethik (16).

Ein realistisches Ziel kann nicht darin bestehen, dass alle Beteiligten schlussendlich zu denselben Einschätzungen gelangen. Der moralische Pluralismus ist eine gesellschaftliche Realität und bleibt auch nach der gründlichen Erkundung schwieriger Themenfelder als solcher bestehen. Wenn die Biound Medizinethik hingegen dazu beitragen kann, dass an die Stelle eines diffusen moralischen Pluralismus ein aufgeklärter Pluralismus ethisch gut begründeter, durchdachter und der Komplexität der Fragestellungen angemessener Meinungen tritt, hat sie einen wichtigen Beitrag zur Aufklärung, moralischen Orientierung und zur Ermöglichung verantwortlichen Handelns in der Gesellschaft geleistet.

Interessenkonflikte: Der Autor bestätigt, dass hinsichtlich des vorliegenden Beitrags keine Interessenskonflikte bestehen.

\section{Abstract}

Loss of Ethics? Bioethics between institutionalisation and critique of ideology

The institutionalisation of biomedical ethics, and its sometimes resulting criticism as ideology are both expressions of social insecurity and dissatisfaction, arising among other things from the rapid - and difficult to grasp - progress of biomedicine, the disapearance of stable value-systems and the orientation they offered, decreased authority of the medical profession, and increased focus on individual autonomy.

The thesis proposed here is that this context threatens to erode thoughtful critical analysis, intelligent weighing of pros and cons on the basis of experience, moral convictions, broadly held values, plausible arguments and ethical theories.

After a brief inventory, this paper presents current examples where the search for ethical answers is interrupted prematurely based on references to applicable law, the autonomy of the affected person, lacking empirical data, required procedures, respected cultural or religious traditions, or concerns about ideology. Finally, it outlines conditions for good bioethics and medical ethics likely to stand the test of time: it will remain important in future to maintain spaces where interdisciplinary search processes based on ethical insights remain possible within controversies. One realistic goal is to aim to replace a diffuse moral pluralism with a more enlightened pluralism of opinions grounded in reason, which could enable moral orientation and responsible action.

\section{Résumé}

\section{Perte de l'éthique? La bioéthique entre institutionalisation et critique des idéologies}

Tant l'institutionalisation de l'éthique biomédicale que sa critique comme idéologie représentent l'expression d'insécurités et d'insatisfactions sociales, consécutives aux progrès rapides et difficiles à comprendre de la biomédecine, à la dissolution de systèmes de valeurs et d'orientation stables, à la perte d'autorité du corps médical et à l'accent sur l'autonomie individuelle. La thèse proposée ici est que ce contexte menace d'éroder la réflexion critique, la pesée intelligente du pour et du contre sur la base de l'expérience, des convictions morales, de valeurs répandues, d'arguments et de théories éthiques plausibles. Après un bref inventaire, cet article présente des exemples actuels d'arrêt prématuré des processus de recherche éthique sur la base d'appels au droit en vigueur, à l'autonomie de la personne affectée, du manque de données empiriques, des procédures applicables, de traditions culturelles ou religieuses respectées, mais aussi de soucis en lien avec la critique des idéologies. Finalement, il esquisse des conditions pour une bioéthique et une éthique médicale justifiables et capables de soutenir le test du temps: il demeurera important à l'avenir de maintenir des espaces dans lesquels des processus interdisciplinaires de recherche basés sur des (re)connaissances éthiques sont possible au sein de controverses. Un but réaliste serait de tendre à remplacer un pluralisme moral diffus par un pluralisme plus éclairé d'opinions fondées en raison, capable de fournir une base à l'orientation morale et à l'action responsable. 


\section{Korrespondenz}

Dr. theol. Markus Zimmermann-Acklin

Departement für Moraltheologie und Ethik

Universität Fribourg

Av. de l'Europe 20

CH-1700 Fribourg

e-mail: markus.zimmermann@unifr.ch

Eingang des Manuskripts: 18.01.2010

Eingang des überarbeiteten Manuskripts: 24.03.2010

Annahme des Manuskripts: 27.03.2010

\section{Referenzen}

1. Teuwsen P. «Dazu sage ich nichts», Interview mit Bundesrat Pascal Couchepin. Die Zeit,11, 5.3.2009.

2. Teuwsen P, Meili M. «Wir finden enttäuschend wenig Sinn», Interview mit Christoph Rehmann-Sutter, dem Präsidenten der Nationalen Ethikkommission. Die Zeit, 12, 12.3.2009.

3. Vattimo G. Vom «naturalistischen Fehlschluss» zur Ethik der Endlichkeit. In: Weiss MG (Hg.). Bios und Zoë. Die menschliche Natur im Zeitalter ihrer Reproduzierbarkeit, Frankfurt a.M.: Suhrkamp 2009, 179-190.

4. Körtner U.H.J. Personen werden geboren. Zur Kritik der Vorstellung von der Person als causa sui und ihren Konsequenzen für die Ethik. In: Weiss MG (Hg.). Bios und Zoë. Die menschliche Natur im Zeitalter ihrer Reproduzierbarkeit, Frankfurt a.M.: Suhrkamp 2009, 240-272.

5. Fischer J. Medizinethik und Moral, in: Bioethica Forum 2006; Nr. 48,8 f.

6. Wiesemann C. Von der Verantwortung, ein Kind zu bekommen Eine Ethik der Elternschaft, München: Beck 2006.
7. Zimmermann-Acklin M. Bioethik in theologischer Perspektive. Grundlagen, Methoden und Bereiche, Freiburg i.Ue., Freiburg i.Br.: Academic Press Fribourg, Herder 2. Auflage 2010.

8. Seel M. Aktive und passive Selbstbestimmung. Merkur 2000; $54: 626-32$.

9. Schicktanz S, Schildmann J. Medizinethik und Empirie Standortbestimmungen eines spannungsreichen Verhältnisses. Ethik in der Medizin 2009;21:183-6.

10. Krones T. Empirische Methodologien und Methoden der angewandten und der empirischen Ethik, in: Ethik in der Medizin 2009;21:247-258.

11. Baumann-Hölzle R, Arn C (Hg.). Ethiktransfer in Organisationen, Basel: Schwabe 2009.

12. Pieper A. Das ausgelagerte Gewissen. Der Boom der Ethikkommissionen. Neue Zürcher Zeitung, 28.3.1998, 81.

13. Blackburn E. Bioethics and the Political Distortion of Biomedical Science. New England Journal of Medicine 2004; 350:1379 -80.

14. Schöne-Seifert B. Induzierte pluripotente Stammzellen: Ruhe an der Ethikfront? Editorial. Ethik in der Medizin 2009; 21:271-273.

15. Birnbacher D. Wieweit lassen sich moralische Normen mit der «Natur des Menschen» begründen? In: Weiss MG (Hg.). Bios und Zoë. Die menschliche Natur im Zeitalter ihrer Reproduzierbarkeit, Frankfurt a.M.: Suhrkamp 2009, 219-239.

16. Autiero A. Verletzender Fundamentalismus. Ein Plädoyer für mehr Besonnenheit in der Stammzelldebatte. Die Zeit, 2, 3.1.2008.

17. Braidotti R. Zur Transposition des Lebens im Zeitalter des genetischen Biokapitalismus, in: Weiss MG (Hg.). Bios und Zoë. Die menschliche Natur im Zeitalter ihrer Reproduzierbarkeit, Frankfurt a.M.: Suhrkamp 2009, 108-135.

18. Gehring P. Was ist Biomacht? Vom zweifelhaften Mehrwert des Lebens, Frankfurt a. M.: Campus 2006.

19. Krones T. Kontextsensitive Bioethik. Wissenschaftstheorie und Medizin als Praxis, Frankfurt a.M.: Campus 2008.

\section{The Fragility of Bioethics}

_Viewpoint

«Bioethics» as a term was still widely unknown some 15 or 20 years ago. Today we can find bioethics issues appearing daily in the press: the birth of octuplets, a parliamentary debate on active euthanasia, the establishment of a new national committee, to mention just a few random examples.

The development of bioethics could be called a success story. There is an increasing number of journals worldwide, many of which continue to rise in visibility and impact. If we displayed the growth of the different ethics commissions, councils and committees on a global scale the curve would probably be quite impressive. Likewise, countless teaching programs and tools have been developed for different curricula, for medical and nursing schools as well as for other settings. Consultation services, both private and public, have been established, and a respectable number of national and international professional societies provide space for networking and exchange.

It is fair to say that bioethics has turned into a flourishing field. Why? For one, it is intellectually fascinating. It is simply fun to think how we should think morally of new forms of life that we designed through the means of synthetic biology, or why we should or should not pop a pill that would turn us into moral whiz kids. And secondly, bioethics has the potential to provide advice and guidance on questions that are of practical relevance and frequently of public concern. Should we aim at a stricter implementation of anti-doping measures or rather at regulation and supervision? What arguments can be put forward for any of the two options? Whereas bioethics as a field 